\title{
The Current Use and the Evolution of Erythrocyte Sedimentation Rate Measurement
}

\author{
Müjdat Aytekin ${ }^{1}$ \\ ${ }^{1}$ University of Halic, Faculty of Medicine, Department of Biochemistry \\ Received: 12 February 2018 Accepted: 31 March 2018, Published online: 20 April 2018 \\ (C) Ordu University Institute of Health Sciences, Turkey, 2018
}

\begin{abstract}
Objective: The golden standard for measuring erythrocyte sedimentation rate (ESR) is the Westergren method. Some other methods for measuring ESR have become available in the recent decades. They differ from a modest modification of the Westergren method to very different methodologies. Even though the methodologies of these tests are different the results of them are assessed as the same test by clinicians. Therefore the bias reasoned by different methodology has caused misinterpretation of the patients by clinicians. The comparison and harmonization of this method will provide a standardization and same interpretation for ESR.

Methods: The golden standard method for measuring ESR which is called Westergren Method and two prototypes of the remaining methods were chosen for comparison. Three different group of patients were were assessed with three different methods in this study.

Results: The monitorization of three samples were observed for 60 minutes. There was no correlation detected depending on the time after the monitorization had started. Hence the adjustment of the results were collected after 20 or 30 minutes for simulating 60 minutes were found significantly different than the classical Westergren method. The detected bias between the adjustment of the earlier result to one hour and the classical method is not always different than the classical method. The sample results were found significantly different in comparison to two methods for all selected patient groups.

Conclusion: There is no sedimentation algorithm detected in ESR monitorization process. Therefore the observed bias is thought to be caused by the adjustment of the earlier results to one hour. The adjusted results of 30 minutes were found to be significantly similar compared to the adjusted results of 20 minutes. The results of the capillary photometric kinetic method were found unbiased with the classical Westergren method and compatible with the clinical observations.

Key words: erythrocyte, sedimentation rate, laboratory hematology, laboratory standards, recommendations, westergren
\end{abstract}

Address for correspondence/reprints:

Müjdat Aytekin

Telephone number: +90 (553) 8800623

E-mail: mujdataytekin@ halic.edu.tr,

DOI: $10.19127 / \mathrm{mbsjohs} .393733$

\section{Introduction}

When well mixed venous blood is placed in a vertical tube, erythrocytes tend to fall down. The meaning of Erythrocyte Sedimentation Rate is measuring the rate of erythrocyte fall or settle in the plasma of a drawn anticoagulated blood specimen over 60 minutes in millimeters/hour (Batlivala 2009; Janson and Tischler, 2012). However it may be measured more quickly in the recently defined ESR methods with centrifugation or other the accelerated sedimentation methods (Janson and Tischler, 2012; Kratz1et al., 2017). ESR is useful but it isn't a specific marker for underlying inflammation. Most recently CRP or other inflammatory markers have 
been used to detect or monitor disease particularly cardiovascular disease and metabolic syndrome (Xia and Samols 1997; Colombet et al., 2010; Litao and Kamat, 2014). The theory of erythrocyte sedimentation was first observed and defined in 1897 by Dr. Edmund Faustyn Biernacki, who found that ESR is varied among individuals and that red blood cells (RBCs) fall more quickly in the presence of increased levels of fibrinogen (Grzybowski and Sak, 2011).

In 1918, Dr. Robert Fahraeus observed that ESR differed in pregnant in comparison to non-pregnant women and recommended the test as a candidate marker for detection of pregnancy. In 1921, Dr. Alf Vilhelm Albertsson Westergren used ESR as a marker in the prognosis of patients with pulmonary tuberculosis. Dr. Westergren described the measurement standards for the ESR test that are still used widely today in which sodium citrate is used as an anticoagulant (Westergren, 1926).

There are lots of factors which cause erythrocyte to be settled faster. Some of these factors are sourced plasma protein and erythrocyte mass is also an important factor which affects ESR.

The ESR can be influenced by a wide variety of factors, including alterations of the quality and quantity of the RBCs, as well as the changes in the normal patterns and the amount of various plasma proteins. Therefore this widely used test is vulnerable to misinterpretation in clinical practice (Olshaker and Jerrard 1997; Jurado, 2001).

Aggregation of erythrocytes causes falling and increases the ESR. RBCs are negatively charged and tend to repel one another in a healthy individuals' whole blood sample. In the presence of positively charged, large and asymmetric acute phase proteins such as fibrinogen and immunoglobulins increases the ESR. An accelerated ESR is accompanied mostly with increase Fibrinogen concentration and a less extent in alpha 2, beta and gamma globulins. These asymmetric protein molecules have a greater effect than the other proteins in decreasing the negative charge of erythrocytes which is called zeta potential. Zeta potential causes to keep the erythrocytes apart. The decreased zeta potential causes erythrocyte rouleaux formation which causes a faster sedimentation. Removal of fibrinogen by defibrination or any other reason causes a lower ESR. There was no absolute correlation between ESR and any plasma protein fractions detected. Red cell is also one of the most important factor which affects ESR. Anemia independently increases the ESR because of increasing rouleaux formation of changes in the concentration of plasma proteins. ESR is proportional to the weight of cell aggregate and inversely proportional to the total surface area of erythrocyte. Microcyte sediment is slower than macrocyte because of smaller total erythrocyte surface area (Olshaker and Jerrard, 1997).

Anemia and polycythemia (primary and secondary) represent quantitative changes in erythrocytes in various clinical conditions and will increase and decrease the ESR, respectively. Similarly, hemoglobinopathies and conditions associated with altered erythrocytes such as sickle cell disease have a low sedimentation rate during sickle crises that increases in the presence of moderate to severe infections (Miller et al, 1983; Ahmed et al, 2000).

ESR is a very simple and may be one of the most used laboratory test over 100 years. Although it is a non-specific marker for inflammation it's still assessed oftenly by clinicians in diagnosis and monitorization of many diseases. Recently there are many new measurement methods developed for measuring ESR. Even though these methods are very similar to the original Westergren method there are some differences in the process of measurement. Some of these methods use centrifugation for getting a faster test result and some use micro sedimentation (Table 1). In some methods ESR is measured in 20th or 30th minutes and adjusted to 60th minute by using a formula (Kratz1 et al, 2017). 
Table 1. Partial listing of ESR instruments and their methodologies

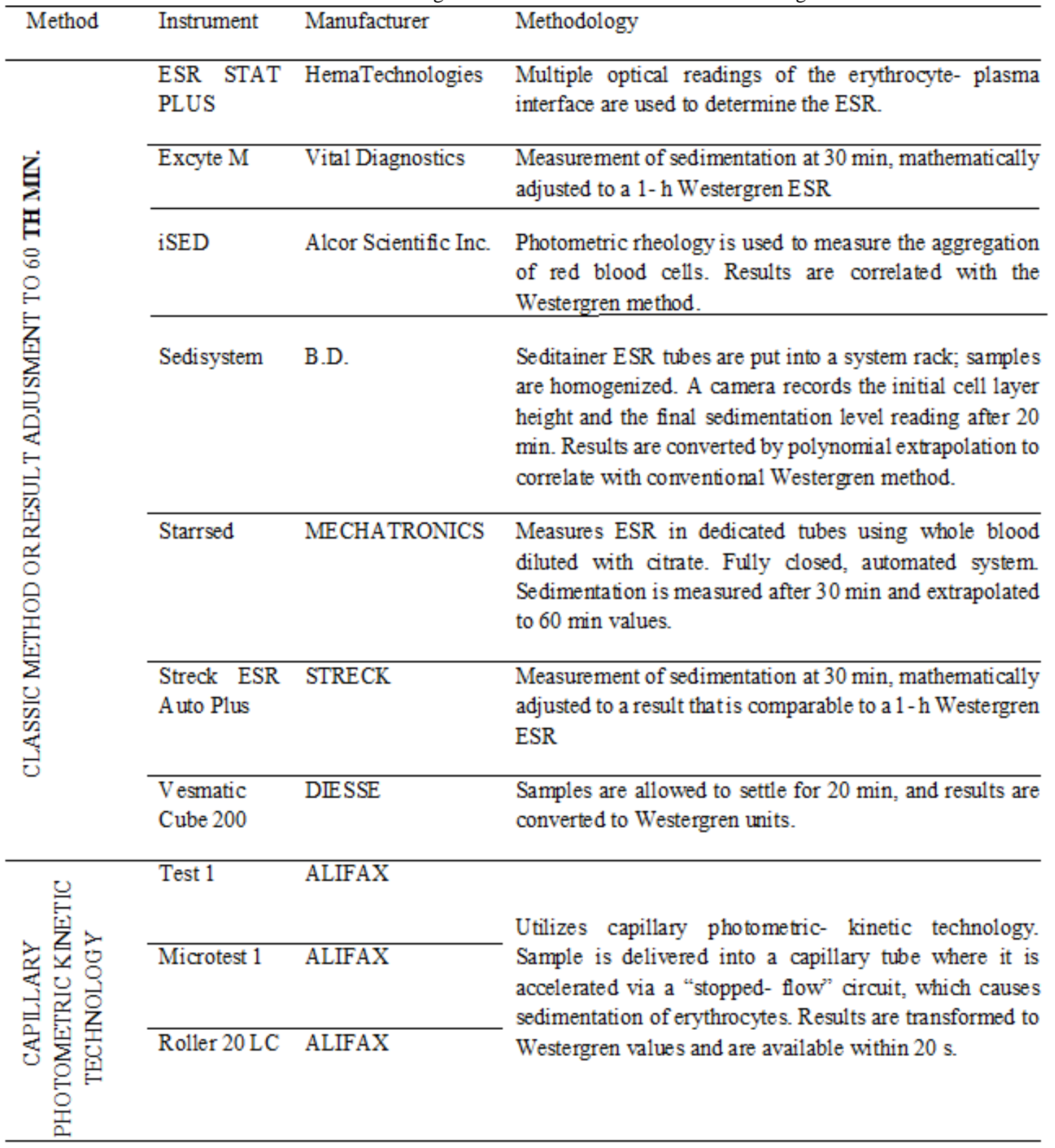

In this study we are aiming to investigate the difference of new methods and compare the results of these methods with the classical Westergren's method. The methods in use for ESR test differ by their methodology of measurement. The methods may be divided into two main groups simply. One of these two groups is the measurement method and it is based on the detection of the sedimentation level in an earlier stage ( 20 or $30 \mathrm{~min}$.) and reflection to the $60^{\text {th }}$ minute by a formula. The other is more sophisticated which is based on the acceleration and the monitorization of the sedimentation. Two instruments were chosen for the study each representing the group given above. And the correlation of the results with the Westergren method were investigated.

\section{Methods}

Blood samples anticoagulated with K2EDTA (Becton Dickinson, Franklin Lakes, NJ) were routinely obtained from hospitalized patients at the Florence Nightingale Caglayan Hospital İstanbul / Turkey processed for analysis. $2 \mathrm{~mL}$ tubes were used in the study including K2EDTA for anticoagulant. 
All samples were obtained under standardized conditions (in the morning after a night of fasting) and tested within 4 hours of venipuncture, according to ICSH recommendations. Between October 2017 and December 2017, we selected 74 blood samples from patients with malignancy $(\mathrm{n}=22)$, autoimmune disease $(n=20)$, or infection $(n=32)$; all patients had TEST 1 ESR values of $20 \mathrm{~mm} / \mathrm{h}$ or more and hematocrit values between $33 \%$ and $40 \%$ (0.330.40) Table 2.

WESTERGREN Method: The Westergren method was performed according to ICSH specifications on undiluted blood samples anticoagulated with K3EDTA using glass pipettes (Greiner Bio-One, Kremsmuenster, Austria). During sedimentation, the pipettes were mounted vertically on appropriate supporting racks and kept at room temperature, which never exceeded $25^{\circ} \mathrm{C}$.

VES MATIC CUBE 200 Method: Samples are initially rotated slowly for 2 minutes and screened for start up volume detection than allowed to settle for 20 minutes, and results are adjusted for 60 minutes to Westergren units.

TEST 1 Method: The TEST 1 analyzer, a closed automated analyzer, determines the duration of sedimentation reaction in blood in a standard-sized primary tube with a perforating stopper. The principle of measurement is the study of the aggregation capacity of RBCs by capillary photometric technology. The tubes are placed in the racks of the instrument, and their contents are rotated slowly for about 2 minutes. By using a closed aspiration needle, the blood is directly drawn from the collection tube, distributed in a capillary, and centrifuged at about $20 \mathrm{~g}$. The system uses an infrared ray microphotometer with a light wavelength of $950 \mathrm{~nm}$ and performs 1,000 readings in 20 seconds. The electrical impulses, collected using a photodiode detector, are directly correlated to the aggregation of RBCs present at each capillary level. For each sample, an aggregation and sedimentation curve is obtained. A mathematical algorithm converts the raw data obtained from evaluation of optical density signals into ESR results, which are transformed to comparable Westergren values. The system operates at a rate of 180 specimens per hour in continuous loading, providing a result every 20 seconds, and requires 150 $\mu \mathrm{L}$ of blood for each sample (Plebani et al., 1998; Plebani and Piva, 2002; Romero et al., 2003; Piva et al., 2007).
Statistical Analysis We compared the correlations of Westergren with TEST 1 ESR and Westergren with VES MATIC CUBE 200 Method results. All statistical analyses were performed using SPSS 13.0 software (SPSS, Chicago, IL).

\section{Results}

The patients test results with Westergren method for 60 minutes who are suffering from infection, malignancy and autoimmunity are seen in the graphic 1-2 and 3 respectively.

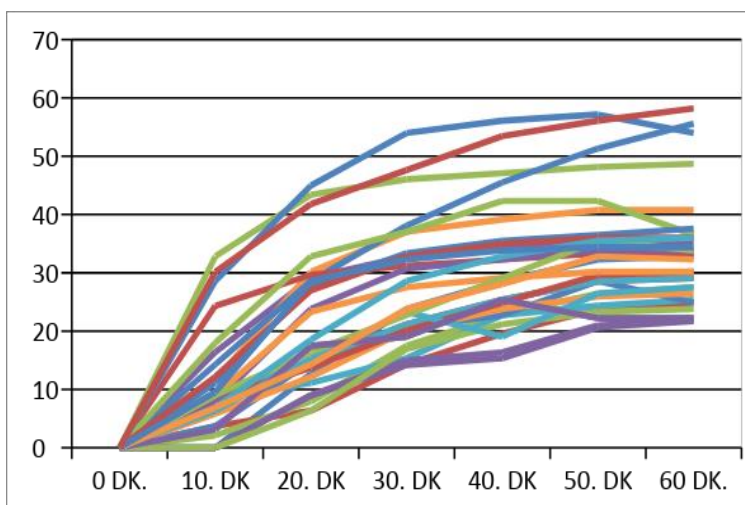

Graphic 1. One hour sedimentation monitorization of the patients samples suffering from infection

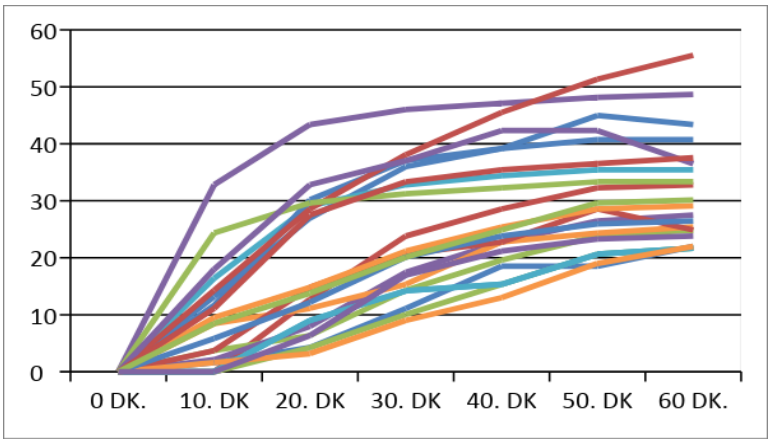

Graphic 2. One hour sedimentation monitorization of the patients samples suffering from malignancy

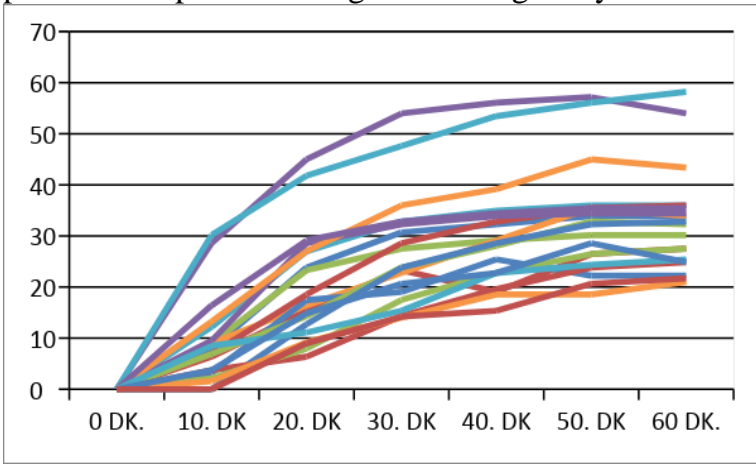

Graphic 3. One hour sedimentation monitorization of the patients samples suffering from autoimmune diseases 


\section{The Evolution of ESR Test}

It can be seen that in graphic 1-2 and 3 there are similar curves measured in 60 minutes for all patient groups. The results of $20^{\text {th }}$ minute and $30^{\text {th }}$ minute are compared with the results of $60^{\text {th }}$ minute in graphic 4 and 5 respectively. The results of $30^{\text {th }}$ minute was found in more correlation with the $60^{\text {th }}$ minutes results rather than the results of $20^{\text {th }}$ minute.

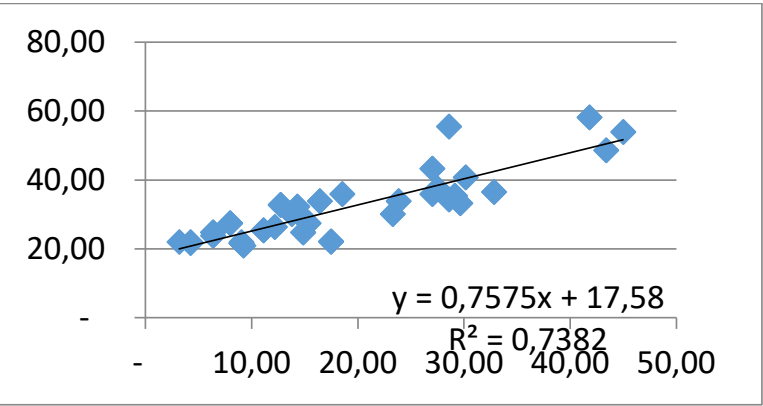

Graphic 4. The results in 20 minutes comparing with the results for $60^{\text {th }}$ minutes

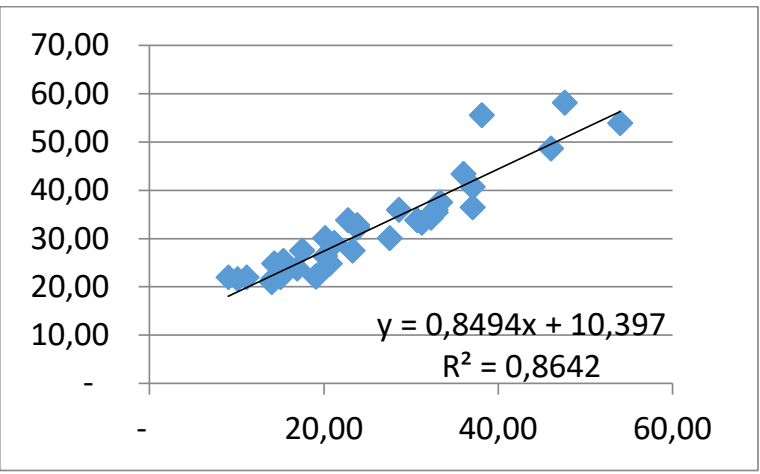

Graphic 5. The results in 30 minutes comparing with the results for $60^{\text {th }}$ minutes

Method comparison of VESMATIC CUBE 200 method with classic Westergren method in the patient group suffering from infection, malignancy and autoimmunity are seen in the graphic 6-8 and 10 respectively. And comparison of TEST 1 method with classic Westergren method in the patient group suffering from infection, malignancy and autoimmunity are seen in the graphic 7-9 and 11 respectively. In all patient groups TEST 1 method was found significantly in correlation with the Westergren method.

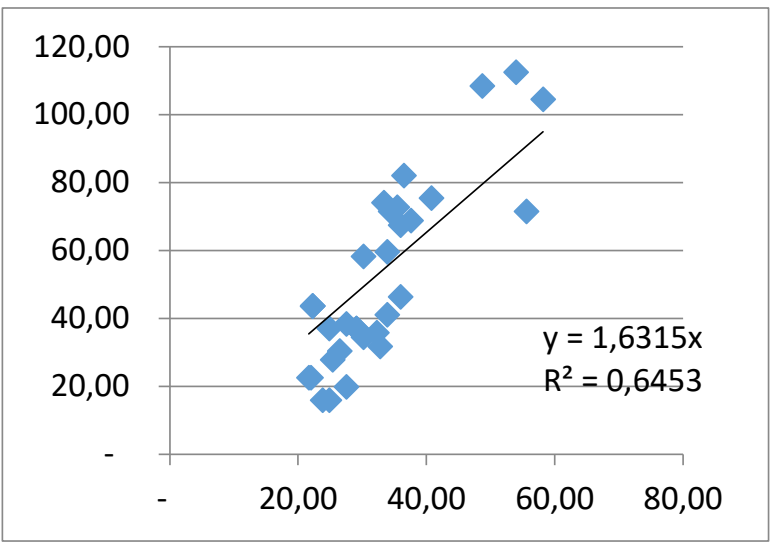

Graphic 6. Regression analysis of Westergren method vs. VESMATIC Cube 200 for patients suffering from infection

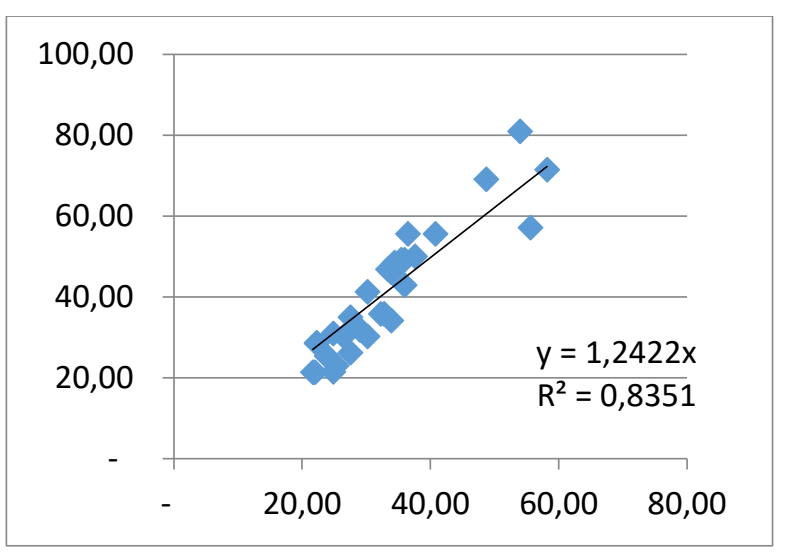

Graphic 7. Regression analysis of Westergren method vs. TEST 1 for patients suffering from infection

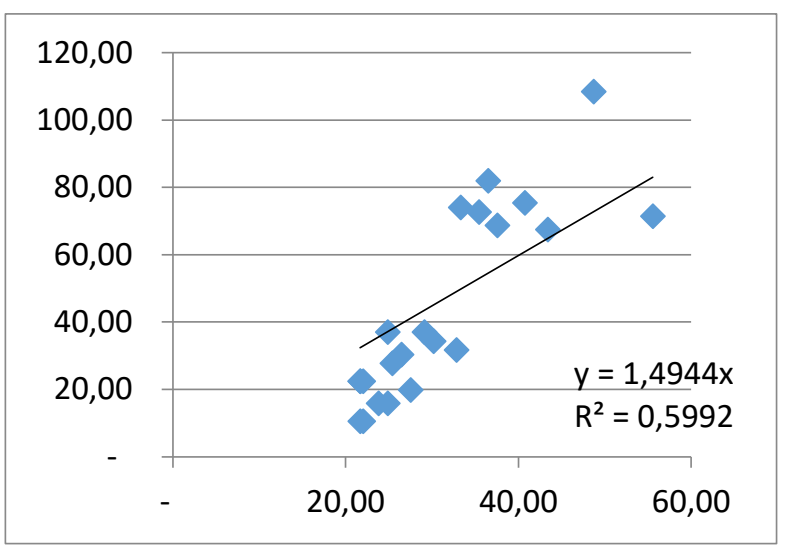

Graphic 8. Regression analysis of Westergren method vs. VESMATIC Cube 200 for patients suffering fr malignancy 


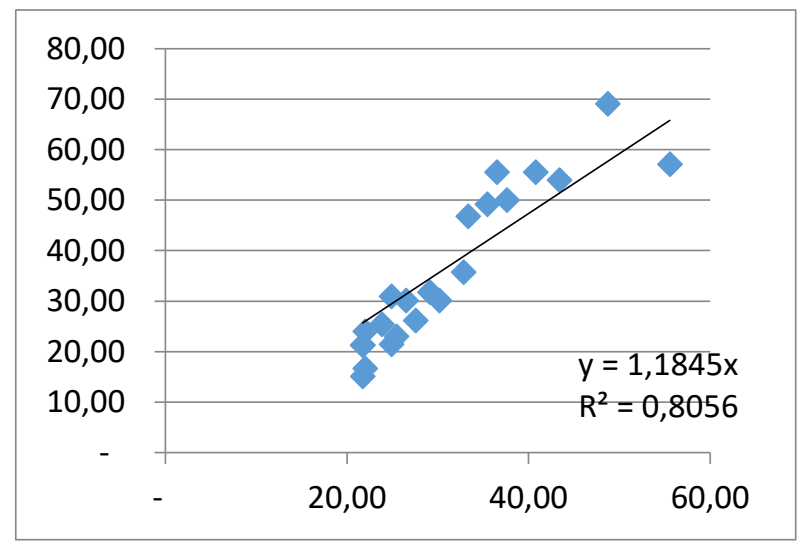

Graphic 9. Regression analysis of Westergren method vs. TEST 1 for patients suffering from malignancy

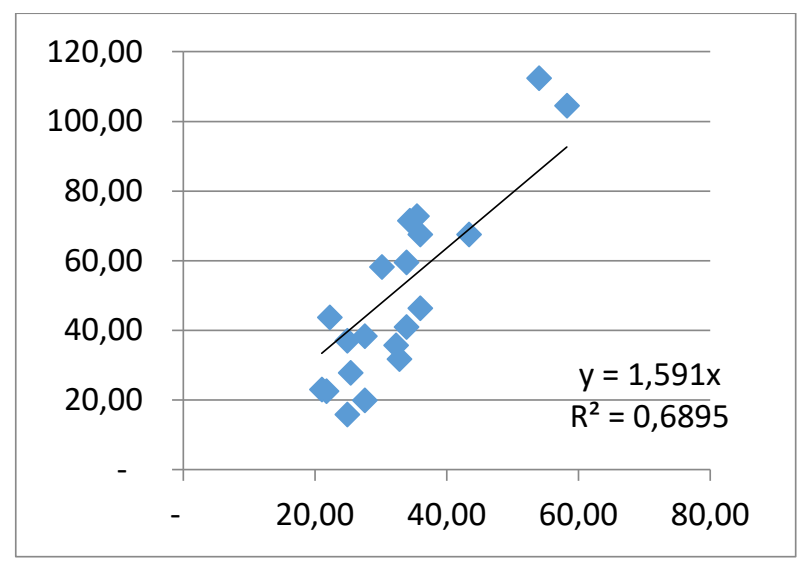

Graphic 10. Regression analysis of Westergren method vs. VESMATIC Cube 200 for patients suffering from autoimmunity

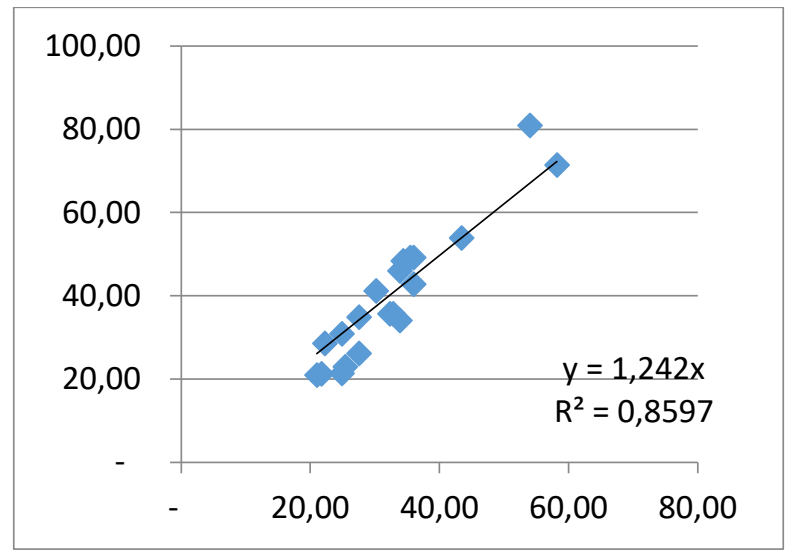

Graphic 11. Regression analysis of Westergren method vs. TEST 1 for patients suffering from autoimmunity

\section{Discussion}

The Westergren method is used as the golden standard method for ESR measurement. Because of the methodology of this measurement, plasma proteins, morphology and quantity of erythrocyte affect the results. This is the most important weakness of the method for reliability and the accuracy of the test result (Batlivala 2009). Therefore clinicians should know the morphology and quantity of erythrocyte mass of a patient while they are assessing the result of ESR test. By the way the results of the instruments which are produced to give faster results are examined by the clinicians. As it is understood from the Graphic 1-2 and 3 the adjusted results from $20^{\text {th }}$ and $30^{\text {th }}$ minutes are not well correlated with the results of the Westergren method. The results adjusted from $30^{\text {th }}$ minute has a better correlation with the Westergren method rather than the results adjusted by using the $20^{\text {th }}$ minutes results. Either Westergren or the other methods in which adjustment of the earlier result is being used are sensitive from the quantity of the erythrocytes in the whole blood. (A. Kratz1, M. Plebani, M. Peng, Y.K. Lee, R. McCafferty, S.J. Machin, 2017) Therefore the patients results are not well reflecting their statement in anemia or policytemia. Eventhough the patient is suffering from infection or malignity their ESR measurements may be lower than expected because of polycythemia or over than expected in the cases accompanying severe anemia. This interaction can be assessed for the weakness of these methods even for the golden standard Westergren method. The instruments in which the Capillary Photometric Kinetic Method are used like TEST 1 measurements of the instrument is performed by an accelerated sedimentation process. In this method the most possible sedimentation level is aimed to be detected by an accelerated sedimentation process and the rate of each samples are measured and converted to the Westergren method. Therefore the result of this method is unaffected from the interference of the quantity and the morphology of erythrocytes like anemia or polycythemia. This specificity is the most important strength of the Capillary Photometric Kinetic Method. Extremely fast result evaluation is also one of the most important strengths of this method. This method provide clinicians a clear results which are not affected by erythrocyte mass or structure.

Even though the measurement of ESR is still a non-specific laboratory test with the new Capillary Photometric Kinetic Method will be kept to be used 
as the most commonly used test without prior weakness of sensitivity which is based on the erythrocyte morphology and quantity.

\section{Conclusion}

Conclusion In this study, westergren method is accepted the golden standart method for E.S.R. measurement since it's first found by Dr. Westergren in the early $20^{\text {th }}$ century with many of the weaknesses in assessment of the patient results during clinical examination. The new Capillary Photometric Kinetic Method is seemed to cover some of the most important weaknesses of the original method. Therefore with the clear explanation of this new method to the clinicians it is believed to be preffered in the diagnosis of inflammation and infection instead of the other similar measurement methods.

Ethics Committee Approval: Approval was received for this study from in Halic University Counseling and Research Center.

Peer-review: Externally peer-reviewed.

Author Contributions: Concept - M.A.; Design M.A.; Supervision- M.A.; Materials - M.A.; Data Collection and/or Processing - M.A.; Analysis and/or Interpretation - M.A..; Literature Review M.A.; Writing M.A.; Critical Review - M.A.

Conflict of Interest: No conflict of interest was declared by the authors.

Financial Disclosure: The authors declared that this study hasn't received no financial support.

\section{References}

Ahmed YF, Abbag FI, Al-Qahtani JM, Ghazali BM, Abolfotouh MA. Erythrocyte sedimentation rate during steady state, painful crisis and infection in children with sickle cell disease. Saudi Med J. 2000; 21(5): 461-463.

Batlivala SP. Focus on diagnosis: the erythrocyte sedimentation rate and the $\mathrm{C}$-reactive protein test. Pediatr Rev. 2009; 30(2): 72-74.

Colombet I, Pouchot J, Kronz V, Hanras X, Capron L, Durieux P, et al. Agreement between erythrocyte sedimentation rate and $\mathrm{C}$-reactive protein in hospital practice. Am J Med. 2010; 123(9): 863.e7-13.

Grzybowski A, Sak JJ. Who discovered the erythrocyte sedimentation rate? J Rheumatol. 2011; 38(7): 1521-1522; author reply 1523 .
Janson L, Tischler M. The Big Picture: Medical Biochemistry. New York, NY: McGrawHill; 2012.

Jurado RL. Why shouldn't we determine the erythrocyte sedimentation rate? Clin Infect Dis. 2001; 33(4): 548-549.

Kratz1, M. Plebani, M. Peng, Y.K. Lee, R. McCafferty, S.J. Machin, ICSH recommendations for modified and alternate methods measuring the erythrocyte sedimentation rate. Int $\mathbf{J}$ Lab Hem. 2017; 39: 448-457.

Litao MK, Kamat D. Erythrocyte sedimentation rate and C-reactive protein: how best to use them in clinical practice. Pediatr Ann. 2014; 43(10): 417420.

Miller A, Green M, Robinson D. Simple rule for calculating normal erythrocyte sedimentation rate. Br Med J (Clin Res Ed). 1983; 286(6361): 266.

Olshaker JS, Jerrard DA. The erythrocyte sedimentation rate. J Emerg Med. 1997; 15(6): 869-874

Piva E, Pajola R, Temporin V. A new turbidimetric standard to improve the quality assurance of the erythrocyte sedimentation rate measurement. Clin Biochem. 2007; 40: 491-495.

Plebani M, De Toni S, Sanzari MC, The TEST 1 automated system: a new method for measuring the erythrocyte sedimentation rate. Am J Clin Pathol. 1998; 110: 334-340.

Plebani M, Piva E. Erythrocyte sedimentation rate: use of fresh blood for quality control. Am J Clin Pathol. 2002; 117: 621-626.

Romero A, Munoz M, Ramirez G. Length of sedimentation reaction in blood: a comparison of the TEST 1 ESR system with the ICSH reference method and the Sedisystem 15. Clin Chem Lab Med. 2003; 41: 232-237.

Westergren A. The technique of the red cell sedimentation rate. Am Rev Tuber. 1926; 14: 94 101.

Xia D, Samols D. Transgenic mice expressing rabbit C-reactive protein are resistant to endotoxemia. Proc Natl Acad Sci USA. 1997; 94(6): 25752580. 